Research Paper

\title{
A Prospective Cohort Study of Biomarkers in Squamous Cell Carcinoma of the Anal Canal (SCCAC) and their Influence on Treatment Outcomes
}

\author{
Camila Motta Venchiarutti Moniz ${ }^{1,2}$, Rachel Pimenta Riechelmann 3 , Suilane Coelho Ribeiro Oliveira ${ }^{4}$, \\ Giovanni Mendonça Bariani ${ }^{1}$, Thomas Giollo Rivelli ${ }^{1}$, Cintia Ortega ${ }^{1}$, Allan Andresson Lima Pereira ${ }^{5}$, \\ Sibele Inácio Meireles ${ }^{6}$, Rejane Franco ${ }^{7}$, Andre Chen ${ }^{1}$, Renata Colombo Bonadio ${ }^{1}$, Caio Nahas ${ }^{1}$, Jorge \\ Sabbaga ${ }^{1}$, Renata Almeida Coudry6,8, Maria Ignez Braghiroli1,2, Paulo Marcelo Hoff ${ }^{1,2}$ \\ 1. Instituto do Cancer do Estado de Sao Paulo (ICESP), Hospital das Clinicas HCFMUSP, Faculdade de Medicina, Universidade de Sao Paulo, Sao Paulo, SP, \\ BR. \\ 2. Instituto $D^{\prime}$ Or de Pesquisa e Ensino - IDOR, Sao Paulo, SP, BR \\ 3. Clinical Oncology Department, AC Camargo Cancer Center, Sao Paulo, BR \\ 4. Faculdade de Ciencias Médicas da Universidade Estadual do Piaui (UESPI), Piaui, BR. \\ 5. Hospital Sírio Libânes, Brasília, BR. \\ 6. Hospital Sírio Libânes, Sao Paulo, BR. \\ 7. Universidade Federal do Paraná - Hospital de Clínicas, Curitiba, PR, Brasil. \\ 8. UnitedHealth Group Brazil.
}

$\triangle$ Corresponding author: Camila Motta Venchiarutti Moniz, E-mail: venchiarutti.c@gmail.com, camila.venchiarutti@hc.fm.usp.br; Phone: 55 11 99644-6112. Address: Av. Dr. Arnaldo, 251 - Cerqueira César, São Paulo - SP, 01246-000. Sao Paulo, Brazil.

(c) The author(s). This is an open access article distributed under the terms of the Creative Commons Attribution License (https://creativecommons.org/licenses/by/4.0/). See http://ivyspring.com/terms for full terms and conditions.

Received: 2020.12.29; Accepted: 2021.10.05; Published: 2021.10.16

\begin{abstract}
Background: Although Chemoradiation (CRT) is the curative treatment for SCCAC, many patients present primary resistance. Since it is a rare tumor, response predictors remain unknown.

Methods: We performed a prospective cohort study to evaluate biomarkers associated with CRT response, progression-free survival (PFS), and overall survival (OS). The primary endpoint was response at 6 months $(\mathrm{m})$. Tumor DNA and HPV were analyzed by next-generation sequencing, while $\mathrm{KI}-67$ and PD-LI by immunohistochemistry in tumor tissue.

Results: Seventy-eight patients were recruited between October/2011 and December/2015, and 75 were response evaluable. The median age was 57 years, $65 \%(n=49)$ were stage III and $12 \%(n=9)$ were HIV positive $(\mathrm{HIV}+)$. At $6 \mathrm{~m}, 62.7 \%(\mathrm{n}=47)$ presented $\mathrm{CR}$. On multivariate analyses, stage Il patients were 4.7 more likely to achieve response than stage III (OR, 4.70; $95 \% \mathrm{Cl}, 1.36-16.30 ; \mathrm{p}=0.015)$. HIV+ was associated with a worse response (OR, 5.72; $95 \% \mathrm{Cl}, 2.5-13.0 ; \mathrm{p}<0.001)$. 5-year PFS and OS rates were $63.3 \%$ and $76.4 \%$, respectively, with a median follow up of $66 \mathrm{~m}$. On multivariate analyses, older age (HR 1.06, $p=0.022,95 \% I C 1.01-1.11)$ and absence of $C R$ at $6 \mathrm{~m}$ (HR 3.36, $\mathrm{p}=0.007,95 \% \mathrm{IC} 1.39-8.09)$ were associated with inferior OS. The 5 -year OS rate was $62.5 \%$ in HIV+ group compared to $78 \%$ among HIV$p t s$, although this difference was not statistically significant $(p=0.4)$. PIK3CA, MET and TP53 mutations, HPV, Ki-67 expression, and PD-Ll expression, were not associated with PFS and OS.
\end{abstract}

Conclusions: Clinical stage III and HIV+ were associated with worse response to CRT at $6 \mathrm{~m}$. The absence of CR was the main factor associated with poor 5-year OS.

Key words: Anal Carcinoma, Biomarkers, Ki-67, PD-L1, HPV, HIV

\section{Introduction}

CRT cures most patients with localized SCCAC. However, some tumors present primary resistance and $15 \%$ do not achieve complete clinical response
(CR) at 26 weeks from CRT onset [1]. Lack of response to CRT is a poor prognostic factor with a 5-year overall survival (OS) rate of $48 \%$ versus $87 \%$ among 
responders [1]. Staging, on its own, is unable to explain all cases of uncontrolled disease, and only a few retrospective studies have been conducted to explore predictive CRT response biomarkers in SCCAC. In the absence of prospective studies in this scenario, stratification of recurrence risk and treatment intensification recommendations are lacking.

Immunosuppression and infection by the Human papillomavirus (HPV) play an important role in SCCAC. The integration of the HPV viral DNA in the host cells and oncoproteins E6/E7 production results in the inactivation of the tumor suppressor gene proteins $\mathrm{pRb}$ and $\mathrm{p} 53$ [2,3], HPV also activates the PI3K/Akt/mTOR pathway, promoting cell proliferation [2]. In terms of prognosis, HPV+, together with p16 positivity, has been associated with better local control at 5 years among SCCAC patients [4]. A recent systematic review and meta-analyses and 17 retrospective cohort studies indicated that $\mathrm{HPV}+$, and P16+, had improved OS [5]. TP53 mutations are described in SCCAC HPV- tumors and PIK3CA mutations were related to poor OS in patients who underwent salvage surgery post local relapse [6].

While HIV positivity is a known risk factor for developing SCCAC, randomized trials of SCCAC have consistently excluded $\mathrm{HIV}+$ patients [7]. Immune-mediated response against $\mathrm{HPV}$ and the tumor involves complex interactions among $\mathrm{T}$ cells, cytokines, and immune checkpoints. However, little is known about the intricacy of immune system operation during HPV, HIV, and tumor subsistence interaction $[8,9]$. The literature on HIV+ SCCAC patient outcomes is scarce and studies about the predictive and prognostic impact of HIV status have reported conflicting results.

The presence of HPV integrated inside the nucleus of tumor cells makes the tumor more immunogenic, but cancer cells may express PD-L1 to neutralize the host immune response. The PD-1/PD-L1 checkpoint can be activated by the PI3K-AKT pathway, inducing upregulation of PD-L1 [10]. Phase II studies demonstrated immunotherapy activity in metastatic SCCAC, but the impact of PD-L1 expression in the primary tumor was unknown during radical treatment with curative intent [11]. Another potential predictive biomarker in SCCAC is the Ki-67 index, as suggested by a systematic review, with a possible association of high Ki-67 with longer disease-free survival (DFS) [12].

Due to the rarity of this disease, the data available about predictive factors in SCCAC come from retrospective series, which are subject to numerous biases, including patients who are not treated with QT/RDT full dose and analyses of tumor samples from different sites and treatment stages.

We aimed at detecting, in a prospective trial, if clinical factors or tumor biomarkers analyzed in the primary tumor before starting treatment (HIV, HPV, PD-L1, Ki-67, and DNA tumor mutations) were associated with CRT efficacy.

\section{Materials and Methods}

Consecutive patients with localized SCCAC at the "Instituto do Câncer do Estado de São Paulo" were invited to participate in this study, from October/2011 to December/2015. Inclusion criteria were histological diagnosis of invasive SCCAC, stage T2-4 N0 M0 or T1-4 N1-3 M0 according to the AJCC VII [13], $\geq 18$ years old, and be candidates for complete treatment with curative intent. Standard treatment includes radiotherapy 45-54Gy plus a single dose of mitomycin $15 \mathrm{mg} / \mathrm{m}^{2}$ on D1 and 5 -fluorouracil continuous infusion $1000 \mathrm{mg} / \mathrm{m}^{2} /$ day on D1-4 and D29-32. In addition, Capecitabine 825 $\mathrm{mg} / \mathrm{m}^{2}$ orally BID could be used during radiotherapy as a substitute for 5-FU. The sample was estimated as 70 patients based on convenience and feasibility.

HIV tests and tumor samples were obtained before CRT started. Clinical examination and imaging with Response Criteria in Solid Tumors (RECIST) v1.1 [14] were performed at 6-8 weeks and at six months (+/-15 days) after CRT to assess response. Chest and abdominal tomography (CT) plus pelvic CT or magnetic resonance image (MRI) were performed during initial staging and follow-up. After six months, patients were followed every three months during the first two years and every six months from the third to fifth. Imaging was performed annually from the first to the fifth year. During CRT, toxicities were graded using the Common Terminology Criteria for Adverse Events, v3.0 [15]. Laboratory tests were performed before D1 and D29 of CRT. We also collected information on age, sex, ECOG, and CRT doseintensity.

Tumor DNA was assessed for integrity by realtime polymerase chain reaction (PCR) using specific primers included in TruSight Tumor 26-Illumina ${ }^{\circledR}$ kit, that sequence specific exons in 26 genes: $A K T 1, A L K$, APC, BRAF, CDH1, CTNNB1, EGFR, ERBB2, FBXW7, FGFR2, FOXL2, GNAS, GNAQ, KIT, KRAS, MAP2K1, MET, MSH6, NRAS, PDGFRA, PIK3CA, PTEN, SMAD4, SCR, STK11, and TP53. Sample preparation followed the manufacturer's guidelines (www. illumina.com). At least $1000 \times$ coverage of each allele and variant frequency $>5 \%$ were considered for analysis. The identification and classification of variants was performed by Variant Studio Software plus manual online database research at cBioPortal (http://www.cbioportal.org/), Clinvar (https:// 
www.ncbi.nlm.nih.gov/clinvar), Varsome (https:// varsome.com) and The Clinical Knowledgebase (https://ckb.jax.org/).

HPV screening was performed by PapilloCheck ${ }^{\circledR}$ kit (Greiner Bio-One $\mathrm{GmbH}$ ) that identifies HPV 6, 11, 16, 18, 31, 33, 35, 39, 40, 42, 44, 55, $45,51,52,53,56,58,59,66,68,70,73,82$. When sample quality was insufficient for PapilloCheck $\AA$, hybridization in situ using the INFORM HPV III Family 16 and INFORM HPV II Family 6 (Ventana ${ }^{\circledR}$ ) were performed to identify HPV 6,11, 16, 18, 31, 33, 35, $39,45,51,52,56,58$ and 66. Immunohistochemical reactions were performed with mouse anti-human Ki-67 clone MIB-1-DAKO® (1:200) and anti-PD-L1 XP E1L3N clone Cell Signaling $®(1: 100)$, according to the manufacturer's guidelines.

The primary study objective was 6 months response rate. Association between biomarkers (HPV status, ki67, PD-L1, mutations) and clinical variables (stage, age, HIV status, and clinically relevant treatment interruption, defined as a pause of more than 7 days in radiotherapy and/or not completing full chemotherapy plan) and response rate (CR and CR plus PR combined) at six months was assessed by univariable and multivariate logistic regression. Twosided $\mathrm{p}$ values $<0.05$ were considered statistically significant in multivariate logistic regression. Comparisons of categorical variables between groups were performed by Fisher's exact test.

Secondary objectives were OS and PFS. OS was calculated from the date of C1D1 treatment until the date of death from any cause. PFS was considered the time from the date of C1D1 treatment to clinical progression, radiological progression, or death from any cause. Patients without the events were censored at the date of the last follow-up. The Kaplan-Meier method was used to estimate survival functions, and survival curves were compared using the log-rank test. Factors associated with OS were evaluated with univariable and multivariate Cox regression. The potential prognostic factors evaluated were age, stage, treatment interruption, HIV status (positive vs. negative), smoking status (yes vs. no), ECOG (2/3 vs. $0 / 1$ ), HPV status (yes vs. no), CR at $6 \mathrm{~m}$ (no vs. yes), KI-67 ( $\geq 50 \%$ vs. $<50 \%$ ), PD-L1 $>1 \%$ (yes vs. no), codon 72 polymorphism in TP53 (presence vs. absence), MET mutations (presence vs. absence), PIK3CA mutations (presence vs. absence). In the Cox multivariate analysis, $p$ values $<0.05$ were considered statistically significant. Stata Software, version 15 (StataCorp, Texas, USA), was used for statistical analyses. Our local Ethics Committee approved the study and all eligible patients signed an informed consent form.

\section{Results}

From October/2011 to December/2015, we recruited 78 patients. Two patients were excluded due to metastatic disease on initial staging in image review and one withdrew from the study. Seventyfive patients were evaluable for response and 68 had tumor tissues available for biomarker evaluation. Four patients were defined as having CR at 6 months based only on clinical examination because three did not have measurable disease at baseline and one did not perform pelvic imaging. Clinical examination included local evaluation anoscopy and digital rectal exam in all patients. All these 4 patients had sustained CR for more than 2 years. The median age was 57 years, 54 patients (72\%) were female, 49 (65\%) presented stage III disease, nine $(12 \%)$ were $\mathrm{HIV}+$, and the majority $(93.3 \%)$ was ECOG 0/1 (Table 1). All pts HIV + included are male, and the majority had stage III (Table 2).

Table 1. Population Characteristics.

\begin{tabular}{|c|c|c|}
\hline \multicolumn{3}{|c|}{ Baseline Characteristics $(n=75)$} \\
\hline Characteristic & $\mathrm{n}$ & $(\%)$ \\
\hline \multicolumn{3}{|l|}{ Sex } \\
\hline Female & 54 & 72 \\
\hline Male & 21 & 28 \\
\hline \multicolumn{3}{|l|}{ HIV Status } \\
\hline Positive & 9 & 12 \\
\hline Negative & 66 & 88 \\
\hline \multicolumn{3}{|l|}{ ECOG } \\
\hline 0 & 34 & 45.3 \\
\hline 1 & 36 & 48 \\
\hline 2 & 4 & 5.3 \\
\hline 3 & 1 & 1.4 \\
\hline \multicolumn{3}{|l|}{ Stage } \\
\hline II & 26 & 34.7 \\
\hline IIIA & 19 & 25.3 \\
\hline IIIB & 30 & 40 \\
\hline \multicolumn{3}{|c|}{ Primary Tumor (T) } \\
\hline $\mathrm{T} 1$ & 1 & 1.4 \\
\hline $\mathrm{T} 2$ & 31 & 41.3 \\
\hline $\mathrm{T} 3$ & 18 & 24 \\
\hline $\mathrm{T} 4$ & 25 & 33.3 \\
\hline \multicolumn{3}{|c|}{ Regional Lymph Nodes (N) } \\
\hline No & 35 & 46.7 \\
\hline N1 & 15 & 20 \\
\hline N2 & 18 & 24 \\
\hline N3 & 7 & 9.3 \\
\hline \multicolumn{3}{|c|}{ Median Age - years (range from) } \\
\hline HIV positive & $50(42 ; 60)$ & \\
\hline HIV negative & $56(38 ; 79)$ & \\
\hline All patients & $57(38 ; 79)$ & \\
\hline
\end{tabular}

At 6-8 weeks after treatment, 41 patients (54.6\%) attained CR, $28(37.3 \%)$ presented partial response (PR), two $(2.7 \%)$ had stable disease, and three $(4 \%)$ experienced progression (PD) as best response. At six months after CRT, 47 (62.7\%) patients achieved CR, 18 (24\%) PR, and 10 (13.3\%) PD only. All patients who hadn't presented either CR or PR at 6-8 weeks, 
developed PD at six months.

At the six months evaluation, the outcomes among the HIV+ $(n=9)$ and HIV- $(n=66)$ groups were, respectively: CR 33\% $(n=3)$ versus $66 \% \quad(n=44)$ $(\mathrm{p}=0.07)$, PR $33 \%(\mathrm{~N}=3)$ versus $22.7 \%(\mathrm{n}=15)(\mathrm{p}=0.44)$, and PD $33 \%(n=3)$ versus $10.6 \%(n=7)(p=0.09)$. All HIV patients with PD received full-planed CRT. The outcomes among the stage II $(n=26)$ and III $(n=49)$ were, respectively: CR $84.6 \% \quad(n=22)$ versus $51 \%$ $(n=25)(p=0.005)$, PR $11.6 \%(N=3)$ versus $30.6 \%(n=15)$ $(\mathrm{p}=0.08)$, and PD 3.8\% $(\mathrm{n}=1)$ versus $18.4 \% \quad(\mathrm{n}=9)$ $(\mathrm{p}=0.15)$.

Table 2. Study Population Characteristics according to HIV Status

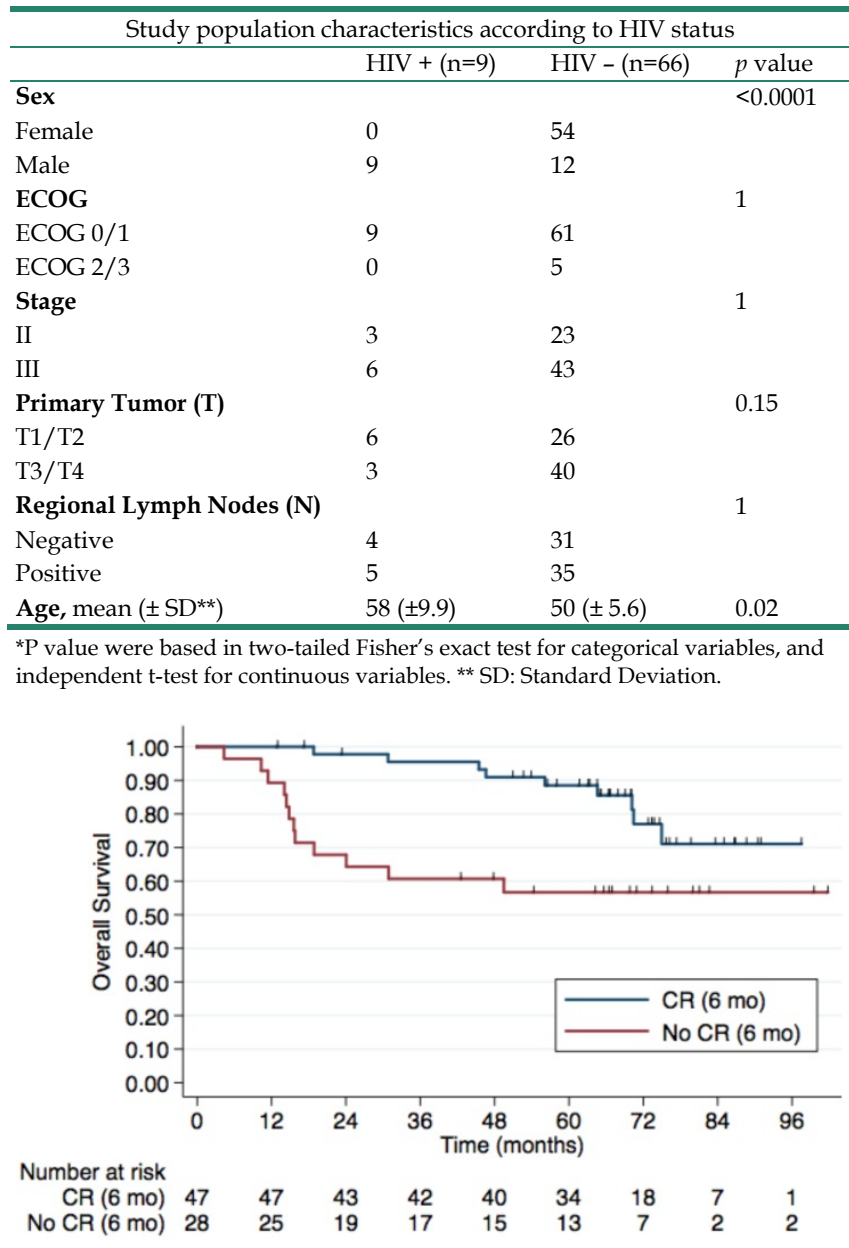

Figure 1. The 5 -year OS rates were $88.5 \%$ in CR group vs. $56.6 \%$ in non-CR group ( $\mathrm{HR} 3.36,95 \% \mathrm{Cl}, 1.39-8.09$; $\mathrm{p}=0.007$ )

The most common grade $3 / 4$ toxicities were lymphopenia in $62(82.6 \%)$ and radiodermatitis in 45 $(60 \%)$ patients. Seventy percent $(\mathrm{N}=53)$ had clinically relevant treatment interruption, mostly due to radiodermatitis. Two patients had febrile neutropenia and no treatment-related deaths occurred.

HPV was evaluated in 67 patients and found in $47(70.1 \%)$, and 45 patients $(60 \%)$ presented high-risk
HPV, considering in situ hybridization and PapilloCheck ${ }^{\circledR}$ evaluation.

It was possible to perform the PapilloCheck ${ }^{\circledR}$ test in 39 patients. Twenty-five had HPV positive samples being the most frequent type was the HPV 16 in 20 patients, followed by HPV 35 in three patients.

PD-L1 was tested in 61 patients, with 10 (16.4\%) being positive ( $>1 \%$ positive expression). Ki-67 was performed in 65 , and the median value was $50 \%$ (range 1-90\%). HIV+ patients exhibited a higher Ki-67 (median 80\%) than HIV- (median 50\%).

On univariable analysis of CR at six months, the stage was associated with $\mathrm{CR}(\mathrm{OR}, 5.28 ; 95 \% \mathrm{CI}$, 1.58-17.59; $\mathrm{p}=0.007)$. On multivariate analysis, stage II patients were 4.7 times more likely to attain CR than those with stage III (OR, 4.70; 95\% CI, 1.36-16.30; $\mathrm{p}=0.015)$. HIV-positive patients had a trend towards lower CR rate (OR, 2.53 95\% CI, 0.9-7.1; $\mathrm{p}=0.079$ ). Considering CR plus PR in a separate multivariate model, HIV+ was significantly associated with lower rates of response (CR plus PR) at six months (OR, 5.72; 95\% CI, 2.5-13.0; $\mathrm{p}<0.001)$. Results of the logistic regression of factors associated with complete response at 6 months are shown in Table 4.

NGS was performed in samples of 27 patients, 25 of whom had a response evaluation. At least one mutation was found in $17(17 / 25,68 \%)$ (Table 3). The most commonly mutated genes were PIK3CA and $M E T$ in six patients each. The mutation described in MET was initially not found in the genomic databases. Later, during the evolution of the cohort, it was described as potentially benign. Due to poor information available in the literature, we chose to keep the MET mutation in the study analyses. Six-month CR rates were similar according to MET mutation status $(50 \%$ in $M E T$-mutated vs. $47.3 \%$ in MET-wild type, $\mathrm{p}=1.0)$; or PIK3CA mutation status (33.3\% in PIK3CA-mutated vs. $47.3 \%$ in PIK3CA-wild type, $\mathrm{p}=0.6)$. The c.215C $>\mathrm{G}$ TP53 polymorphism was found in $72 \%$ (18 of the 25 patients who were tested) and wasn't associated with CR either (44\% in those with TP53 codon 72 polymorphism vs. $57 \%$ in those without TP53 codon 72 polymorphism, $\mathrm{p}=0.6$ ). Among patients who did not carry polymorphisms $(n=7)$ none presented PD at 6 months, while PD at 6 months occurred in $33 \%(n=6)$ of those with the polymorphism.

After a median 66-month follow-up of the 75 patients, 21 deaths occurred. In multivariate analyses, older age, and absence of complete response at 6 months were associated with inferior PFS and OS. The 5 -year OS rates were $88.5 \%$ in CR group vs. $56.6 \%$ in non-CR group (HR 3.36, 95\% CI, 1.39-8.09; p=0.007) (Figure 1). Univariable and multivariate analyses of factors associated with OS are shown in Table 5. 
Table 3. 27 samples were submitted to molecular analyses and 25 patients had response evaluation by RECIST after QRT treatment. HPV can be identified by type on samples suitable to the PapilloCheck $₫$ - patients who performed only in situ hybridization show positive or negative results for the presence of HPV.

\begin{tabular}{|c|c|c|c|c|c|c|c|c|c|}
\hline \multicolumn{10}{|c|}{ Mutations in SCCAC (N=27) } \\
\hline Gene & Exon & Variant & Nucleotide Change & Protein predictive change & Mutation type & Classification & HIV & HPV & Sample \\
\hline$A P C$ & 15 & $\mathrm{C}>\mathrm{C} / \mathrm{T}$ & c. $3985 \mathrm{C}>\mathrm{T}$ & p.His1329Tyr & Missense & Unknown Significance & - & +HPV16 & 48 \\
\hline$B R A F$ & 11 & $\mathrm{C}>\mathrm{C} / \mathrm{T}$ & c. $1379 \mathrm{G}>\mathrm{A}$ & p.Gly460Glu & Missense & Unknown Significance & - & + & 42 \\
\hline $\mathrm{CDH1}$ & 12 & $\mathrm{G}>\mathrm{G} / \mathrm{A}$ & c. $1849 \mathrm{G}>\mathrm{A}$ & p.Ala617Thr & Missense & Benign & + & +HPV16 & 30 \\
\hline FBXW7 & 10 & $\mathrm{C}>\mathrm{C} / \mathrm{T}$ & c. $1420 \mathrm{G}>\mathrm{A}$ & p.Val474Ile & Missense & Unknown Significance & - & +HPV16 & 38 \\
\hline FBXW7 & 10 & $\mathrm{C}>\mathrm{C} / \mathrm{T}$ & c. $1550 \mathrm{G}>\mathrm{A}$ & p.Gly517Glu & Missense & Unknown Significance & - & +HPV16 & 05 \\
\hline FBXW7 & 12 & $\mathrm{G}>\mathrm{G} / \mathrm{A}$ & c. $1972 C>T$ & p.Arg658Ter & Stop Gain & Presumed Pathogenic & - & +HPV35 & 31 \\
\hline KRAS & 5 & $\mathrm{C}>\mathrm{C} / \mathrm{T}$ & c. $564 \mathrm{G}>\mathrm{A}$ & p.Met188Ile & Missense & Unknown Significance & - & +HPV16 & 48 \\
\hline MET & 2 & $\mathrm{C}>\mathrm{C} / \mathrm{T}$ & c. $1075 \mathrm{C}>\mathrm{T}$ & p.Arg359Ter & Stop Gain & Unknown Significance & - & + & 24 \\
\hline MET & 2 & $\mathrm{C}>\mathrm{C} / \mathrm{T}$ & c. $818 \mathrm{C}>\mathrm{T}$ & p.Thr273Ile & Missense & Unknown Significance & + & - & 32 \\
\hline MET & 2 & $\mathrm{G}>\mathrm{G} / \mathrm{A}$ & c. $457 \mathrm{G}>\mathrm{A}$ & p.Asp153Asn & Missense & Unknown Significance & + & +HPV16 & 36 \\
\hline MET & 2 & $\mathrm{~T}>\mathrm{T} / \mathrm{A}$ & c. $607 \mathrm{~T}>\mathrm{A}$ & p.Ser203Thr & Missense & Likely Benign & - & +HPV16 & 48 \\
\hline MET & 21 & $\mathrm{TC}>\mathrm{TC} / \mathrm{T}$ & c.4058delC & p.Arg1354GlyfsTer17 & Frameshift & Unknown Significance & - & +HPV16 & 51 \\
\hline MET & 2 & $\mathrm{G}>\mathrm{G} / \mathrm{T}$ & c. $504 \mathrm{G}>\mathrm{T}$ & p.Glu168Asp & Missense & Likely Benign & + & - & 76 \\
\hline NRAS & 4 & $\mathrm{G}>\mathrm{G} / \mathrm{A}$ & c. $304 \mathrm{C}>\mathrm{T}$ & p.Arg102Ter & Stop Gain & Unknown Significance & - & +HPV16 & 16 \\
\hline PDGFRA & 18 & $\mathrm{G}>\mathrm{G} / \mathrm{A}$ & c. $2458 \mathrm{G}>\mathrm{A}$ & p.Ala820Thr & Missense & Unknown Significance & - & +HPV16 & 36 \\
\hline PIK3CA & 9 & $G>G / A$ & c. $1633 \mathrm{G}>\mathrm{A}$ & p.Glu545Lys & Missense & Pathogenic & - & +HPV16 & 01 \\
\hline PIK3CA & 21 & $\mathrm{~A}>\mathrm{A} / \mathrm{T}$ & c. $3140 \mathrm{~A}>\mathrm{T}$ & p.His1047Leu & Missense & Pathogenic & + & +HPV16 & 36 \\
\hline PIK3CA & 21 & $\mathrm{~A}>\mathrm{A} / \mathrm{G}$ & c. $3140 \mathrm{~A}>\mathrm{G}$ & p.His1047Arg & Missense & pathogenic & - & +HPV16 & 39 \\
\hline PIK3CA & 9 & $\mathrm{G}>\mathrm{G} / \mathrm{A}$ & c. $1645 \mathrm{G}>\mathrm{A}$ & p.Asp549Asn & Missense & Pathogenic & - & + & 57 \\
\hline PIK3CA & 9 & $G>G / A$ & c. $1645 \mathrm{G}>\mathrm{A}$ & p.Asp549Asn & Missense & Pathogenic & - & +HPV16 & 31 \\
\hline PIK3CA & 9 & $\mathrm{C}>\mathrm{C} / \mathrm{A}$ & c. $1636 \mathrm{C}>\mathrm{A}$ & p.Gln546Lys & Missense & Pathogenic & - & +HPV35 & 74 \\
\hline PTEN & 7 & $\mathrm{C}>\mathrm{C} / \mathrm{T}$ & c. $733 \mathrm{C}>\mathrm{T}$ & p.Gln245Ter & Stop Gain & Pathogenic & + & +HPV16 & 30 \\
\hline TP53 & 9 & $\mathrm{TC}>\mathrm{TC} / \mathrm{T}$ & c.970delG & p.Asp324MetfsTer21 & Frameshift & Pathogenic & - & +HPV16 & 48 \\
\hline TP53 & 8 & $\mathrm{G}>\mathrm{G} / \mathrm{A}$ & c. $817 \mathrm{C}>\mathrm{T}$ & p.Arg273Cys & Missense & Pathogenic & + & +HPV 06 & 25 \\
\hline TP53* & 7 & $\mathrm{C}>\mathrm{C} / \mathrm{T}$ & c. $733 \mathrm{G}>\mathrm{A}$ & p.Gly245Ser & Missense & Pathogenic & - & - & 78 \\
\hline
\end{tabular}

* Mutation in a patient not evaluable for response.

Table 4. In logistic regression - multivariate analysis, HIV positive is significantly associated with less chance of response (CR or PR) at 6 months post definitive chemoradiation.

Factors associated with 6-month response rate by Logistic regression

\begin{tabular}{lllllll}
\hline & \multicolumn{3}{l}{ Univariable Analysis } & \multicolumn{3}{c}{ Multivariate Analysis } \\
\cline { 2 - 7 } & OR & p & $95 \%$ CI & OR & p & 95\% CI \\
\hline Age (continuous variable) & 1.02 & 0.57 & $0.95-1.09$ & & & \\
Stage (III vs. II) & 5.62 & 0.11 & $0.67-47.1$ & 6.517 & 0.080 & $0.80-53$ \\
HIV (positive vs negative) & 4.2 & 0.07 & $0.85-20.7$ & 5.720 & $<0.001$ & $\mathbf{2 . 5 1 - 1 3 . 0}$ \\
HPV (positive vs negative) & 1.48 & 0.61 & $0.31-6.9$ & & \\
Ki-67 ( $\geq 50 \%$ vs < 50\%) & 0.98 & 0.4 & $0.95-1.07$ & & \\
PD-L1 > 1\% (yes vs no) & 1.02 & 0.98 & $0.10-9.83$ & & \\
Treatment Interruption & 0.96 & 0.9 & $0.22-4.12$ & & \\
(yes vs no) & & & & &
\end{tabular}

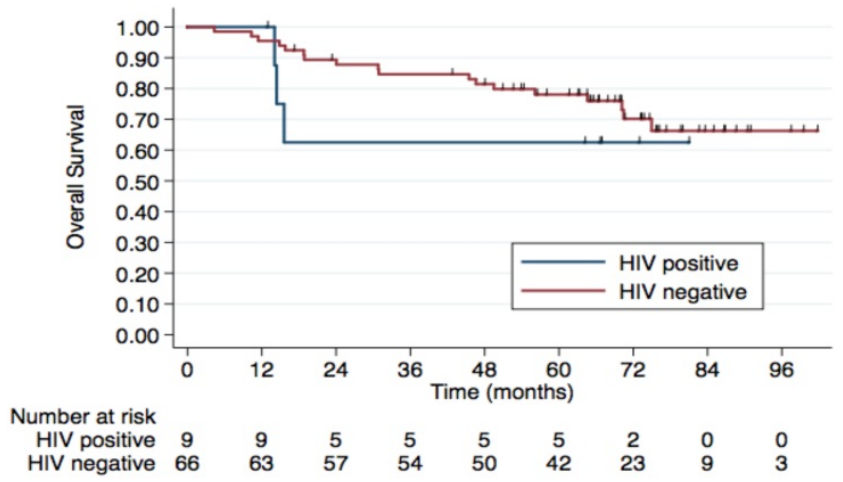

Figure 2. 5-year Overall Survival by HIV Status. The 5-year OS rates were $78 \%$ in the HIV-group vs. $62.5 \%$ in the HIV+ group, although this difference was not statistically significant $(p=0.4)$.
Regarding HIV status, the 5-year PFS rates were $64.7 \%$ in HIV-negative patients and $53.3 \%$ in those HIV-positive. The 5-year OS rates were $78 \%$ and $62.5 \%$, respectively, although this difference was not statistically significant $(p=0.4) \quad$ (Figure 2). No difference was observed according to HPV status either (Figure 3). Patients without TP53 codon 72 polymorphism presented 5-year PFS $71.4 \%$ vs. $37 \%$ and 5-year OS 71.4 vs. $47.4 \%(p=0.181)$ (Figure 4).

Table 5. In multivariate analyses, older age and absence of complete response at 6 months were associated with inferior OS.

Factors associated with overall survival (Cox regression)

\begin{tabular}{|c|c|c|c|c|c|c|}
\hline & \multicolumn{3}{|c|}{ Univariable Analysis } & \multicolumn{3}{|c|}{$\begin{array}{l}\text { Multivariate } \\
\text { Analysis }\end{array}$} \\
\hline & HR & $\mathrm{p}$ & $95 \% \mathrm{CI}$ & HR & $\mathrm{p}$ & $95 \% \mathrm{CI}$ \\
\hline Age (continuous variable) & 1.04 & 0.046 & $1.00-1.09$ & 1.06 & 0.022 & 1.01-1.11 \\
\hline Stage (III vs. II) & 0.93 & 0.883 & $0.38-2.25$ & & & \\
\hline HIV (positive vs negative) & 1.69 & 0.400 & $0.49-5.76$ & & & \\
\hline Smoking (yes vs no) & 1.45 & 0.482 & $0.51-4.16$ & & & \\
\hline ECOG (2-3 vs $0-1)$ & 3.09 & 0.072 & $0.90-10.58$ & & & \\
\hline HPV (positive vs negative) & 0.77 & 0.603 & $0.28-2.05$ & & & \\
\hline Ki-67 ( $\geq 50 \%$ vs $<50 \%)$ & 1.10 & 0.832 & $0.42-2.85$ & & & \\
\hline PD-L1 > 1\% (yes vs no) & 0.38 & 0.351 & $0.05-2.88$ & & & \\
\hline MET mutation (yes vs no) & 2.08 & 0.234 & $0.62-7.01$ & & & \\
\hline PIK3CA mutation (yes vs no) & 1.98 & 0.267 & $0.59-6.62$ & & & \\
\hline c. $215 \mathrm{C}>\mathrm{G}$ TP53 polymorphism & 2.83 & 0.181 & $0.61-13.02$ & & & \\
\hline
\end{tabular}
(yes vs no)

Absence of Complete Response at $6 \mathrm{~m} \quad 2.95 \quad 0.014 \quad 1.24-7.02 \quad 3.36 \quad 0.007 \quad \mathbf{1 . 3 9 - 8 . 0 9}$ (yes vs no)

Treatment Interruption (yes vs no) $\quad 0.91 \quad 0.843 \quad 0.36-2.26$ 


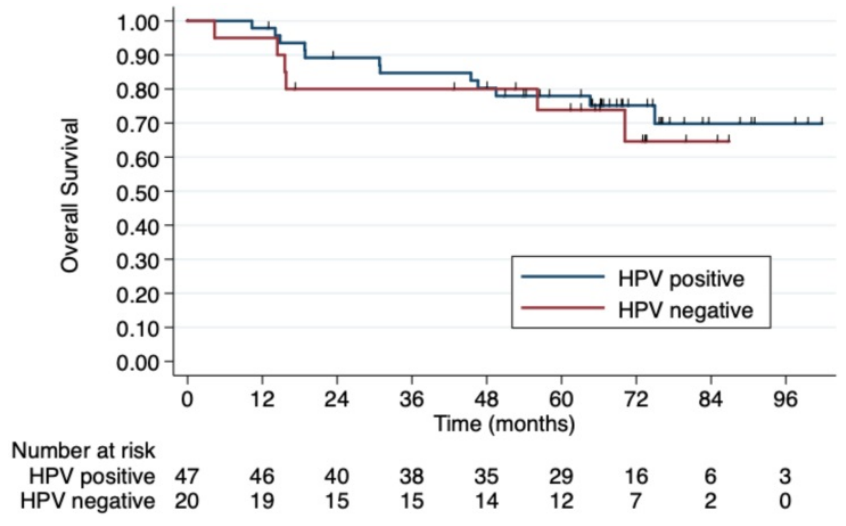

Figure 3. 5-year overall survival by HPV status. No difference in OS was observed according to HPV status.

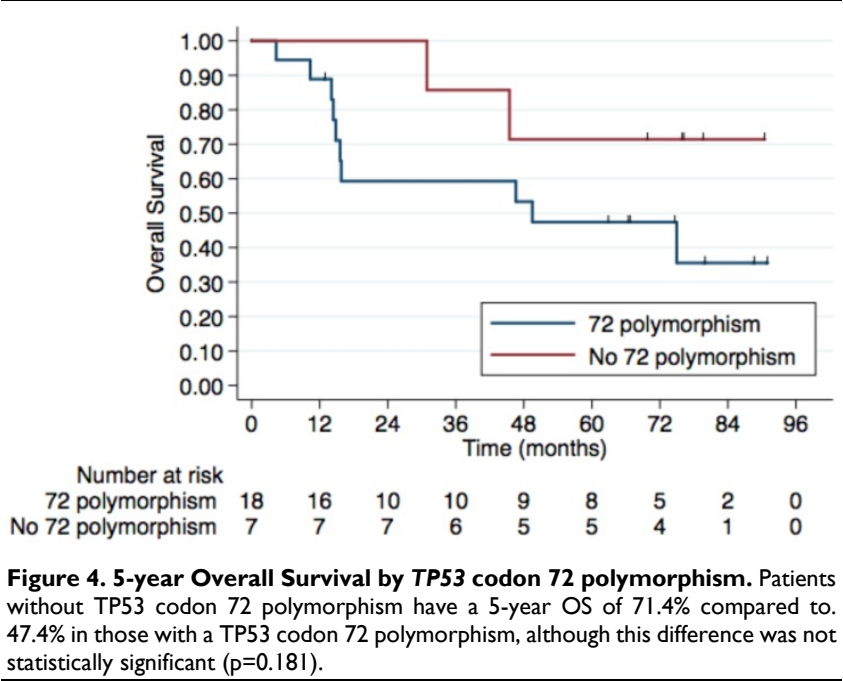

\section{Discussion}

Our prospective cohort study showed that most patients had HPV+ and stage III tumors, and 12\% were HIV positive. Our CR rate at 6 months was $62.7 \%$ lower than observed in the mitomycin arm in ACT II trial at 26 weeks (90.5\%). In ACT II, around $50 \%$ of patients had T1/T2 disease and $63 \%$ node negative disease [7]. Our population had a high number of stage III bulky disease. Our stage II patients had a significantly better response to treatment, with $84.6 \% \mathrm{CR}$ at six months.

Retrospective series showed heterogeneous results, some with complete response rates similar to historical controls in HIV negative (HIV-) [16] and others with lower OS in the HIV+ group [17]. Our population had $12 \%$ HIV positive, and this study demonstrates that HIV is an important biomarker, influencing response to CRT. Only one-third of HIV+ patients achieved $\mathrm{CR}$ at six months, contrasting with $66.7 \%$ of the HIV- group. Also, in the multivariate analysis, HIV- patients were 5.7 times more likely to achieve CR or PR than HIV+. Although HIV status was not associated with survival, CR at 6 months was associated with better PFS and OS in a multivariate model.

Our tumor samples had $70.1 \% \mathrm{HPV}+$, in agreement with the literature that showed HPV in $78 \%$ of SCCAC, the majority HPV16 [18]. In our study, HPV did not significantly influence CR, PFS and OS when prospectively evaluated together with other relevant factors. One possibility is that retrospective cohorts evaluated in the available metanalyses had selection biases and confounders that influenced their results [5].

Ki-67 protein is a nuclear antigen found in proliferating cells [12]. In Ajani [19] study, Ki-67 had significant association with DFS and response. In our study, the ki-67 index did not influence $C R$ at six months (OR, 0.9; 95\% CI, 0.9-1; $\mathrm{p}=0.18$ ). In previous studies, the HIV status was not evaluated systematically and may have been an occult information bias. In our setting, Ki-67 was not a useful marker to predict response, PFS or OS.

In order to evade antitumor immunity $\mathrm{T}$ cell mediated pathway, some tumor cells produce the programmed cell death-ligand 1 (PD-L1) and reduce T cells activation through bonding at a down regulated receptor, PD-1, on surface $\mathrm{T}$ cells [20-22]. A study suggested that $50 \%$ of SCCAC had PD-L1 expression, but $31 \%$ of samples were from recurrent tumors [8]. Immunotherapeutic drugs emerged as a promising treatment in refractory metastatic SCCAC, showing an objective response in $24 \%$ [22]. In a retrospective analysis of 41 tumor samples, PD-L1 positive was associated with poor recurrence rates [23]. In our study, $16.4 \%$ of tumor samples had PD-L1 positive expression and did not influence CR at six months. All our samples were obtained from the primary tumors and prior to CRT, which may explain our different results.

PIK3CA is the most frequent tumor alteration in SCCAC, reported in up to $40 \%$ of patients [24]. While we did not observe a difference in CR according to PIK3CA mutations, our sample was small and not powered to evaluate differences in response rates. The phase I study with Taselisib, a PI3K pathway inhibitor, demonstrated activity in H1047 mutated tumors [25]. In our study, six patients were carriers of PIK3CA mutation, two in H1047 (Table 4), revealing the potential use of PI3K inhibitors in SCCAC. MET pathway crosstalk with PIK3/AKT signaling and MET mutations may carry the cell to a metastatic phenotype. In our study, MET mutations were frequent (24\%) but did not predict CR. Smaglo [26] observed only one mutation in 57 samples. METtargeted therapies, including crizotinib, cabozantinib, capmatinib, tepotinib and glesatinib are being investigated in patients with MET mutations [27]. 
A retrospective study performed in 148 patients observed TP53 mutations in $4.7 \%$, the majority among HPV negative (HPV-) samples. In our study, three patients had TP53 mutation, only one HPV- (Table 3). The E6 HPV oncoprotein interacts with p53 and causes its degradation, and the presence of the germline polymorphism TP53 c. $215 \mathrm{C}>\mathrm{G}$ may increase it [28-30]. Homozygote's individuals showed a 7-fold increased risk of HPV related cancers [31]. We found TP53 c. $215 \mathrm{C}>\mathrm{G}$ in $72 \%$ of tumor samples, and it was not associated with CR at 6 months (44\% vs. 57\%, $\mathrm{p}=0.6)$. However, an intriguing fact was that at six months, no progression was observed among patients without polymorphism. At 5-year follow-up, patients without TP53 c. $215 C>$ G presented better PFS and OS, but this difference was not statistically significant in this cohort.

We observed low mutation rates in other relevant genes (Table 3) described in solid tumors, in agreement with the literature: BRAF $0 \%, K R A S$ in $0-4 \%$, EGFR $0-3 \%[32,33]$.

Our study had limitations, it was a single-center study and the quality/size of the tumor samples permitted molecular studies in only $38 \%$. On the other hand, all patients were systematically treated and followed. A single experienced radiologist and pathologist reviewed, respectively, all the images and biopsy. To our knowledge, this is the first study to analyze biomarkers prospectively in SCCAC.

\section{Conclusion}

This cohort study suggests that HIV- patients had 5.7 times more chance of achieving CR or PR than HIV+ at six months post CRT. Clinical stage and $\mathrm{HIV}+$ were associated with worse response to CRT at $6 \mathrm{~m}$.

The absence of complete response at 6 months was the main factor associated with poor 5-year OS, and new strategies of follow-up and complementary approaches should be studied in these patients to ensure curative treatment success.

In this study, HPV, PIK3CA or MET mutations, Ki-67, and PD-L1 expression did not influence response rate, PFS, and OS. The TP53 c.215C $>$ G polymorphism may potentially predict PFS and OS curves and need to be evaluated in larger prospective.

\section{Implications for practice}

In this prospective study, HIV- patients had 5.7 times more chance of achieving CR or PR than HIV+ at six months post CRT. Considering 5-year OS, the absence of complete response at six months was the main factor associated with a poor prognosis.

Special attention is required for the patient's $\mathrm{HIV}+$ or without a complete response at six months post CRT. It's time to develop and study new strategies for follow-up and complementary approaches in these populations.

\section{Abbreviations}

CR: complete response; CRT: Chemoradiotherapy; HIV: human immunodeficiency virus; HPV: human papillomavirus; $\mathrm{m}$ : months; PD-L1: programmed cell death-ligand 1; PR: partial response; SCCAC: Squamous Cell Carcinoma of the Anal Canal; OS: Overall survival; PFS: progression free survival.

\section{Acknowledgements}

Guilherme Lopes Yamamoto, Carla Daniele de Godoy, Luisa Lina Villa and Ana Carolina Chaves.

\section{Funding}

This work was supported by "Fundação de Amparo à Pesquisa do Estado de São Paulo" [2013/03093-0].

\section{Competing Interests}

The authors have declared that no competing interest exists.

\section{References}

1. Glynne-Jones R, Sebag-Montefiore D, Meadows HM, et al. Best time to assess complete clinical response after chemoradiotherapy in squamous cell carcinoma of the anus (ACT II): a post-hoc analysis of randomised controlled phase 3 trial. Lancet Oncol. 2017; 18(3): 347-56.

2. Zhang L, Wu J, Ling MT, et al. The role of the PI3K/Akt/mTOR signalling pathway in human cancers induced by infection with human papillomaviruses. Mol cancer. 2015; 14: 87 .

3. Bernardi MP, Ngan SY, Michael M, et al. Molecular biology of anal squamous cell carcinoma: implications for future research and clinical intervention. Lancet Oncol. 2015;16(e): 611-21.

4. Mai S, Welzel G, Ottstadt M, et al. Prognostic relevance of HPV infection and p16 overexpression in squamous cell anal cancer. Int J Radiat Oncol Biol Phys. 2015; 93(4): 819-27.

5. Parwaiz I, MacCabe TA, Thomas MG, et al. A systematic review and metaanalysis of prognostic biomarkers in anal squamous cell carcinoma treated with primary chemoradiotherapy. Clin Oncol (R Coll Radiol). 2019; 31(12): e1-e13.

6. Cacheux W, Rouleau E, Briaux A, et al. Mutational analysis of anal cancers demonstrates frequent PIK3CA mutations associated with poor outcome after salvage abdominoperineal resection. Br J Cancer. 2016; 114(12):1387-94.

7. James RD, Glynne-Jones R, Meadows HM, et al. Mitomycin or cisplatin chemoradiation with or without maintenance chemotherapy for treatment of squamous-cell carcinoma of the anus (ACT II): a randomised, phase 3, open-label, 2 × 2 factorial trial. Lancet Oncol. 2013; 14(6): 516-24.

8. Yanik EL, Kaunitz GJ, Cottrell TR, et al. Association of HIV status with local immune response to anal squamous cell carcinoma: implications for immunotherapy. JAMA Oncol. 2017; 3(7): 974-978.

9. Dyck L, Mills KHG. Immune checkpoints and their inhibition in cancer and infectious diseases. Eur J Immunol. 2017; 47(5): 765-779.

10. Martin D, Rodel F, Balermpas P, et al. The immune microenvironment and HPV in anal cancer: Rationale to complement chemoradiation with immunotherapy. Biochim Biophys Acta Rev Cancer. 2017; 1868(1): 221-230.

11. Ott PA, Piha-Paul SA, Munster P, et al. Safety and antitumor activity of the anti-PD-1 antibody pembrolizumab in patients with recurrent carcinoma of the anal canal. Ann Oncol. 2017; 28(5): 1036-41.

12. Lampejo T, Kavanagh D, Clark J, et al. Prognostic biomarkers in squamous cell carcinoma of the anus: a systematic review. Br J cancer. 2010; 103(12): 1858-69.

13. Edge SB, Byrd DR, Compton CC, et al. AJCC Cancer Staging Manual 7th. Chicago, USA: Springer. 2010.

14. Eisenhauer EA, Therasse P, Bogaerts J, et al. New response evaluation criteria in solid tumours: revised RECIST guideline (version 1.1). Eur J Cancer. 2009; 45(2): 228-47. 
15. Trotti A, Colevas AD, Setser A, et al. CTCAE v3.0: development of a comprehensive grading system for the adverse effects of cancer treatment. Seminars in radiation oncology. 2003; 13(3): 176-81.

16. Fraunholz IB, Haberl A, Klauke $\mathrm{S}$, et al. Long-term effects of chemoradiotherapy for anal cancer in patients with HIV infection: oncological outcomes, immunological status, and the clinical course of the HIV disease. Dis Colon Rectum. 2014; 57(4): 423-31.

17. Grew D, Bitterman D, Leichman CG, et al. HIV infection is associated with poor outcomes for patients with anal cancer in the highly active antiretroviral therapy era. Dis Colon Rectum. 2015; 58(12): 1130-6.

18. Hoots BE, Palefsky JM, Pimenta JM, et al. Human papillomavirus type distribution in anal cancer and anal intraepithelial lesions. International journal of cancer. 2009; 124(10): 2375-83.

19. Ajani JA, Wang X, Izzo JG, et al. Molecular biomarkers correlate with disease-free survival in patients with anal canal carcinoma treated with chemoradiation. Digestive diseases and sciences. 2010; 55(4): 1098-105.

20. Welters MJ, de Jong A, van den Eeden SJ, et al. Frequent display of human papillomavirus type 16 E6-specific memory T-helper cells in the healthy population as witness of previous viral encounter. Cancer research. 2003; 63(3): 636-41.

21. de Jong A, van Poelgeest MI, van der Hulst JM, et al. Human papillomavirus type 16-positive cervical cancer is associated with impaired CD4+ T-cell immunity against early antigens E2 and E6. Cancer research. 2004; 64(15): 5449-55.

22. Morris VK, Salem ME, Nimeiri $\mathrm{H}$, et al. Nivolumab for previously treated unresectable metastatic anal cancer (NCI9673): a multicentre, single-arm, phase 2 study. Lancet Oncol. 2017; 18(4): 446-453.

23. Govindarajan R, Gujja S, Siegel ER, et al. Programmed Cell Death-Ligand 1 (PD-L1) Expression in Anal Cancer. Am J Clin Oncol. 2018; 41(7): 638-642.

24. Chung JH, Sanford E, Johnson A, et al. Comprehensive genomic profiling of anal squamous cell carcinoma reveals distinct genomically defined classes. Ann Oncol. 2016; 27(7): 1336-41.

25. Juric D, Krop I, Ramanathan RK, et al. Phase I Dose Escalation Study of Taselisib (GDC-0032), an Oral PI3K Inhibitor, in Patients with Advanced Solid Tumors. Cancer discovery. 2017; 7(7): 704-715.

26. Smaglo BG, Tesfaye A, Halfdanarson TR, et al. Comprehensive multiplatform biomarker analysis of 199 anal squamous cell carcinomas. Oncotarget. 2015; 6(41): 43594-604.

27. Reungwetwattana $\mathrm{T}$, Liang $\mathrm{Y}, \mathrm{Zhu} \mathrm{V}$, et al. The race to target MET exon 14 skipping alterations in non-small cell lung cancer: The Why, the How, the Who, the Unknown, and the Inevitable. Lung cancer. 2017; 103: 27-37.

28. Tan SC, Ankathil R. Genetic susceptibility to cervical cancer: role of common polymorphisms in apoptosis-related genes. Tumour Biol. 2015; 36(9): 6633-44.

29. Beaudenon S, Huibregtse JM. HPV E6, E6AP and cervical cancer. BMC biochemistry. 2008; 9 (Suppl 1): S4.

30. Brady CS, Duggan-Keen MF, Davidson JA, et al. Human papillomavirus type 16 E6 variants in cervical carcinoma: relationship to host genetic factors and clinical parameters. The Journal of general virology. 1999; 80(12): 3233-40.

31. Storey A, Thomas M, Kalita A, et al. Role of a p53 polymorphism in the development of human papillomavirus-associated cancer. Nature. 1998; 393(6682): 229-34.

32. Martin V, Zanellato E, Franzetti-Pellanda A, et al. EGFR, KRAS, BRAF, and PIK3CA characterization in squamous cell anal cancer. Histology and histopathology. 2014; 29(4): 513-21.

33. Paliga A, Onerheim R, Gologan A, et al. EGFR and K-ras gene mutation status in squamous cell anal carcinoma: a role for concurrent radiation and EGFR inhibitors? British Journal of Cancer. 2012; 107(11): 1864-8.

\section{Author Biography}

Camila Motta Venchiarutti Moniz is a clinical oncologist and obtained her PhD degree in Sciences from the University of Sao Paulo Medical School, in 2017. She is currently Medical Manager of clinical trials, and her research is centered on gastrointestinal cancer and rare diseases. 